\title{
Intervention diverses
}

Pierre Desplanques, Pilar Benajam-i-Arguimbau, Clara Dematteis, Tony Male et Antoine Bailly

\section{(2) OpenEdition}

\section{Journals}

Édition électronique

URL : http://journals.openedition.org/trema/2287

DOI : 10.4000/trema.2287

ISSN : 2107-0997

Éditeur

Faculté d'Éducation de l'université de Montpellier

Édition imprimée

Date de publication : 1 mai 1994

Pagination : 81-83

ISSN : 1167-315X

Référence électronique

Pierre Desplanques, Pilar Benajam-i-Arguimbau, Clara Dematteis, Tony Male et Antoine Bailly,

«Intervention diverses », Tréma [En ligne], 5 | 1994, mis en ligne le 01 mai 1994, consulté le 19 avril 2019. URL : http://journals.openedition.org/trema/2287 ; DOI : 10.4000/trema.2287

Ce document a été généré automatiquement le 19 avril 2019.

Trema 


\title{
Intervention diverses
}

\author{
Pierre Desplanques, Pilar Benajam-i-Arguimbau, Clara Dematteis, Tony \\ Male et Antoine Bailly
}

\section{Pierre Desplanques.}

1 J'ai relevé que les questions qui se posent dans les trois pays évoqués et le nôtre sont bien les mêmes. On pourrait déterminer un certain nombre de thèmes. J'en ai personnellement un sur la mise en place des programmes.

2 Ils indiquent ce que nous avons à enseigner, et toute réflexion part de là. Ils l'ont indiqué souvent en termes de contenus, découpage spatial. La conception des programmes estelle fondée sur un découpage spatial, des thèmes géographiques, des notions, des concepts, ou des méthodes? Monsieur le professeur Antoine Bailly est associé à cette question, comme ancien président du baccalauréat international.

\section{Pilar Benajam i Arguimbau.}

3 Chaque pays a un système éducatif propre, ce qui rend les comparaisons difficiles. En Espagne, pas de géographie à l'école primaire, ni dans le secondaire avant 16 ans parce qu'on l'a intégrée dans les sciences sociales (avec l'histoire, le droit, l'économie, la sociologie). La géographie apparaît dans le secondaire supérieur comme option sauf dans le baccalauréat en sciences humaines. On a bien peu de géographie, mais une réforme est en cours avec trois parties d'égale importance : la première sur les concepts qu'on doit expliquer. Roger Brunet serait désespéré, ils commencent par la géographie physique, la population, les travaux des hommes et je suis d'accord avec lui mais il reste à convaincre le gouvernement, ce qui est toujours difficile. 


\section{Clara Dematteis.}

4 En Italie la géographie apparaît dès le primaire, et puis dans le secondaire, toujours de la géographie enseignée non par des spécialistes mais par des généralistes. Le professeur de géographie travaille seulement dans les Instituts techniques, de 14 à 18 ans. Dans la future réforme la géographie sera encore moins enseignée en quantité parce que le généraliste s'en occupera jusqu'à 13 ans. Ensuite, sous forme de cours spécialisés optionnels. La qualité devrait s'améliorer car désormais on va traiter la géographie par problèmes, et non selon un découpage administratif comme dans les anciens programmes. Le professeur pouvait passer aussi directement aux problèmes. Maintenant ce sont de grands thèmes : la population mondiale, l'environnement, l'agriculture, traités avec des exemples régionaux. Mais nous avons encore, en ce moment, le découpage traditionnel par régions.

\section{Tony Male.}

5 Pour l'avenir un groupe de spécialistes très variés réfléchit. C'est autour des Key stages et des Tasks que le débat se concentre en Angleterre. Tous les syndicats ont voté contre, on s'attend à des difficultés. La prise de position entre le Ministre (un enseignant) et les syndicats mettra l'accent sur les problèmes. Suit une lecture des programmes d'études, en anglais (Key stages 3 et Key stages 4, entre 12 et 16 ans), à travers laquelle émergent un certain nombre de concepts que l'on retrouve chez nous.

\section{Antoine Bailly.}

Le baccalauréat international, depuis une dizaine d'années, mène une série d'expériences pour le renouvellement de la géographie. Ce baccalauréat est né dans les années 1950, destiné à des écoles voulant bénéficier d'une éducation internationale. Le problème est très simple, 56 pays sont plus ou moins affiliés à ce baccalauréat, donc dès le départ la géographie pose un problème quelque peu délicat. Vouloir poser la dimension civique (la mienne) aboutirait à une impasse. D'où des objectifs très différents dès le départ. Dans les programmes les décisions ont été minimales, sans éprouver le besoin d'un programme national ce qui serait impossible et irait à l'encontre des programmes "régionationalistes » qui existent çà et là : cantons suisses, submergés par les Alémaniques. Une dimension idéologique qui se retrouve dans d'autre pays.

7 Par contre ce baccalauréat devrait se doter d'un enseignement en géographie susceptible d'être véhiculé dans la cinquantaine de pays quels qu'ils soient, islamiques, américanisés. Les gros problèmes ont été entre Français et Anglais, ce qui n'est pas nouveau, la construction de l'Europe ayant été semée d'embûches. Lors de mon passage à ce baccalauréat nous avons réactualisé le programme, en particulier celui des deux dernières années menant au baccalauréat. Comme en Angleterre il y a un Key stage de 12-16 ans et 16-18 ans qui mène à l'examen.

8 Les structures sont simples, en dehors des contraintes nationales et donc sans syndicats ni sans Etats sur le dos. Les groupes de pression sont multiples et subtils, des écoles ont leur autonomie avec un chef d'établissement qui engage ses professeurs; elles se fédèrent 
entre elles pour monter des programmes : réunion une fois par an, avec des délégués à l'origine d'un examen arrivé à maturité en 1990. Ses grandes trames reflètent la trame conceptuelle puisque l'on ne peut s'entendre ni sur des pays ni sur une série d'éléments régionalistes. On doit donc trouver des clés d'entrée qui soient nouvelles, sans contrainte nationale.

Le programme des $12-16$ et 16-18 progresse en spirale à travers une série de concepts répartis en quatre :

- problème simple : la terre et moi (pour reprendre le titre du livre de Luc Bureau) pour mettre les élèves en situation de se localiser dans le monde, en utilisant une série d'instruments géographiques dont la carte est l'un d'entre eux mais pas le seul : un apprentissage de base pour comprendre les structures du monde telles qu'elles sont représentées.

- pour se substituer à la géographie physique traditionnelle, on a l'environnement sous l'angle (c'est une provocation, en l'absence de Monsieur le Recteur Blanchet) d'une géographie physique intelligente, qui réintroduit l'homme comme élément central de la problématique de la géographie physique, l'homme comme percevant les unités physiques: on ne parle plus de crue mais d'inondation, avec une série de concepts qui permettent de comprendre cette géographie physique.

- les lois et les modèles. Il est nécessaire de les enseigner, et je me sens très proche de Roger Brunet. On connaît depuis bien longtemps la théorie des lieux centraux, et la gravitation. Ce sont des points fondamentaux à enseigner.

- les représentations. J'en suis un fervent représentant et pense que c'est la relativité de la connaissance géographique qui nous permet de valoriser certains éléments.

Ce programme permet, selon le choix des élèves, de se placer en option forte ou en option moyenne parmi les six manières à prendre.

\section{Pierre Desplanques.}

11 Notre gratitude à nos collègues voisins pour ce tableau. Nous sommes en France des privilégiés car l'enseignement va, à quelques exceptions près (enseignement professionnel ou technologique) de l'école primaire à la terminale sans interruption avec un horaire suffisant.

12 En feuilletant Les Mots de la Géographie je me suis arrêté à "géographie », ce qui semble évident. J'y ai lu qu'elle était enseignée par les historiens, ce qui m'a poussé à demander à un historien, doyen de l'Inspection Générale de surcroît, de bien vouloir ouvrir le débat par un exposé sur l'objet de colloque : niveaux d'enseignement et concepts. 
Le point de vue économique : La France est un pays riche de ses chevaux, de ses ânes et de ses mulets.

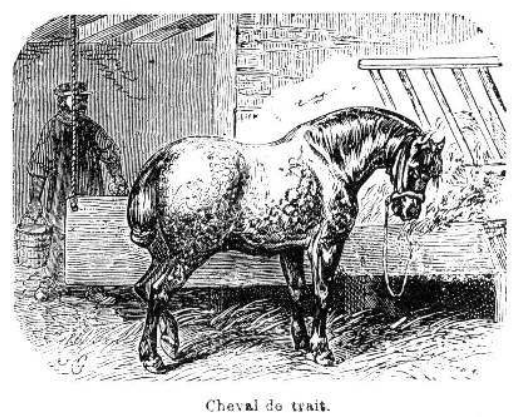

Chevaux, environ $1,500,000$;

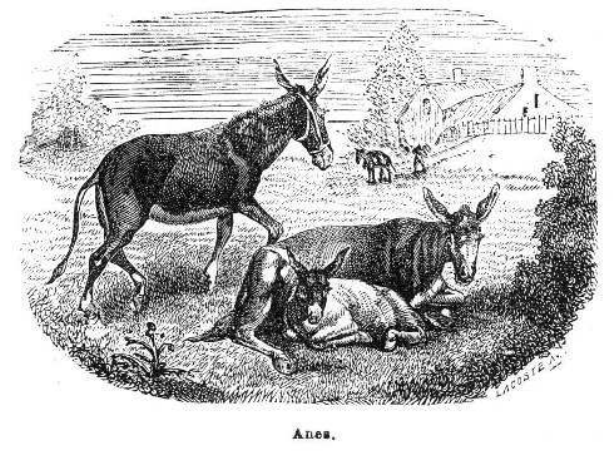

Anes et mulets, 800.000

R. Bravard, de la Bruyère : Nouvelle France pittoresque (Paris, s. e., vers 1872) Géographie nationale (II)

\section{AUTEURS}

\section{PIERRE DESPLANQUES}

Intervenant

\section{PILAR BENAJAM-I-ARGUIMBAU}

Professeur à l'université de Barcelone

\section{CLARA DEMATTEIS}

Chargée de cours à l'université de Turin, spécialiste de didactique de géographie au sein de l' Association des professeur d'histoire et géographie d'Italie

\section{TONY MALE}

Directeur du Central Bureau for Educational Visits \& Exchanges 
ANTOINE BAILLY

Professeur à l'université de Genève 